\title{
Assistência de enfermagem ao paciente com delirium na unidade de terapia intensiva: uma revisão integrativa
}

RESUMO | OBJETIVO: Avaliar o nível de conhecimento do enfermeiro na identificação do Delirium na UTI. METODOLOGIA: Trata-se de uma revisão integrativa, na base de dados SciELO, LILACS e BDENF em março/abril de 2018. Foi dividido em categorias acerca do Delirium, tais como: Reconhecimento do Delirium, Papel da Enfermagem na Utilização da Escala CAM-ICU e Aplicação nas UTIs e Medidas preventivas pelo enfermeiro. RESULTADOS: Para coleta de dados, utilizou-se um instrumento sobre à identificação do artigo, autor (es), periódico publicado, ano da publicação, metodologia do artigo e país de publicação na totalidade de 10 produções. CONCLUSÃO: A dificuldade na identificação da patologia existe, uma vez, que a mesma é subvalorizada e de difícil reconhecimento. Percebemos como uma prática complexa e minuciosa com alguns pontos de fragilidade no que se refere a percepção do Delirium pela equipe multidisciplinar com a finalidade de evitar agravos ao quadro clínico do paciente.

Palavras-chaves: Delirium; Unidade de Terapia Intensiva; Enfermagem.

\begin{abstract}
OBJECTIVE: Assess the level of knowledge of nurses in the identification of Delirium in the ICU. METHODOLOGY: this is an integrative review in SciELO, LILACS and BDENF in March/April 2018. Was divided into categories about the of the Delirium, such as: Recognition of Delirium, and the Role of Nursing in the use of the Scale CAM-ICU and the ICUs and preventive Measures by the nurse. RESULTS: For data collection we used an instrument on the identification of the article, the author (s) journal published, year of publication, methodology of the article and country of publication in all 10 productions. CONCLUSION: The difficulty in identifying the pathology once exists is that it is undervalued and difficult to recognize. We perceive as a complex and meticulous practice with some fragility points regarding the perception of Delirium by the multidisciplinary team with the purpose of avoiding damages to the clinical picture of the patient.
\end{abstract}

Keywords: Delirium; Intensive Care Unit; Nursing.

RESUMEN I OBJETIVO: Evaluar el nivel de conocimiento del enfermero en la identificación del Delirium en la UTI. METODOLOGÍA: Se trata de una revisión integrativa, en la base de datos SciELO, LILACS y BDENF en marzo/abril de 2018. Se dividieron en categorías acerca del Delirium, tales como: Reconocimiento del Delirium, Papel de la Enfermería en la Utilización de la Escala CAM-ICU y Aplicación en las UTIs y Medidas preventivas por El enfermero. RESULTADOS: Para la recolección de datos se utilizó un instrumento sobre la identificación del artículo, autor (es), periódico publicado, año de la publicación, metodología del artículo y país de publicación en la totalidad de 10 producciones. CONCLUSIÓN: La dificultad en la identificación de la patología existe, una vez, que la misma es subvalorizada y de difícil reconocimiento. Percibimos como una práctica compleja y minuciosa con algunos puntos de fragilidad en lo que se refiere a la percepción del Delirium por el equipo multidisciplinario con la finalidad de evitar agravios al cuadro clínico del paciente.

Descriptores: Delirium; Unidad de Terapia Intensiva; Enfermería.

Juliana Gois

Enfermeira. Pós graduanda em enfermagem na Alta Complexidade. Universidade Castelo/ UCB. Rio de Janeiro (RJ). Brasil.

\section{Taiane Vieira}

Enfermeira. Pós graduada em enfermagem na Alta Complexidade. Universidade Castelo Branco/UCB. Rio de Janeiro (RJ). Brasil.

\section{Bruno da Silva Lourenco}

Enfermeiro. Especialista em Terapia Intensiva pela Escola de Enfermagem Anna Nery/EEAN/UFRJ. Docência do Ensino Superior Universidade Candido Mendes. Mestrado Enfermagem Psiquiátrica/EEAN/ UFRJ. Enfermeiro do Setor de Hemodiálise do HFB. Docente na Universidade Estácio de Sá. Rio de Janeiro. (RJ). Brasil.

Recebido em: 17/05/2019

Aprovado em: 26/05/2019

\section{Daniel Ribeiro Soares de Souza}

Enfermeiro. Mestre em saúde e tecnologia no espaço hospitalar - UNIRIO. Enfermeiro coordenador da unidade de nefrologia HUCFF/UFRJ. Docente de Enfermagem na Universidade Castelo Branco e Faculdade São José.

\section{Luana Valentim}

Enfermeira. Mestre em Enfermagem pela Universidade Federal do Rio de janeiro, Escola de Enfermagem Anna Nery. Docente na Universidade Estácio de Sá. Especialista em Terapia intensiva pela Universidade Veiga de Almeida.

\section{Lidiane Reis \\ Enfermeira. Especialista em Saúde da Família e MBA em Gestão em Saúde pela Universidade Gama Filho/RJ. Mestrado em Enfermagem EEAN/UFRJ. Docente Auxiliar e Pós-Graduação Lato Sensu Estácio de Sá- RJ. Docente do Estado FAETEC- RJ. Coaching Acadêmico em Saúde.}

INTRODUÇÃO

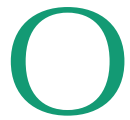
estudo pela temática partiu do interesse de investigar o que está por trás das alterações neurológicas que acometem o paciente internado na Unidade de Terapia Intensiva (UTI). Sendo assim, identificar o que leva ao Delirium tornou-se um desafio para nós.

A patologia apresentada requer uma atenção diferenciada, visto que é uma condição cada vez mais comum entre pacientes hospitalizados, obrigando-nos a ampliar os conhecimentos com relação às ações realizadas na rotina de uma UTI, proporcionando ao paciente acometido por tal patologia o aumento da expectativa de vida.

A abordagem do paciente deve incluir a identificação dos fatores precipitantes e definir intervenções ade- 
quadas, visando a resolução do quadro que pode se apresentar de diferentes formas entre os pacientes.

Desde 500 a.C, Hipócrates já usava diversos termos que descreviam uma síndrome com início agudo de alterações comportamentais, distúrbios do sono e déficits cognitivos, associada à febre, usando mais comumente o termo "frenite", também utilizado como sinonímia para delirium ${ }^{(1)}$.

Mesmo sendo uma doença que, muitas vezes, não é reconhecida pela equipe médica, alguns estudos ${ }^{(1,2)}$ têm demonstrado que a mesma representa em torno de 14 a 24\% de prevalência no momento da admissão. O papel do enfermeiro é de grande importância na identificação precoce do delirium devido à maior quantidade de horas dedicadas ao cuidado com o paciente em UTI.

Delirium é definido como um distúrbio ou flutuação da condição mental, acompanhada de falta de atenção ou alteração dos níveis de consciência ou ainda, um pensamento desorganizado; esse distúrbio neurológico tem recebido pouca atenção dos profissionais que trabalham na UTI, principalmente, por não ser a razão primária da internação ${ }^{(3)}$.

É necessário o conhecimento sobre este distúrbio, com bases cientificas, o reconhecimento dos sintomas facilita a aplicabilidade de medidas e intervenções necessárias, minimizando sua incidência.

O mecanismo de fisiopatologia causador do delirium não é bem compreendido. Porém, seus tipos são bem determinados, como: hiperativo, quando os pacientes se apresentam agitados e inquietos, e hipoativos quando apresentam apatia, letargia, diminuição da atividade motora e de falta de atenção. Sua relevância não se deve somente à elevada incidência, mas, sobretudo, por estar relacionada aos piores prognósticos, alta mortalidade e aos maiores gastos gerados pelo prolongamento da hospitalização. E se o paciente apresentasse uma combinação de ambos os tipos, diagnosticava-se delirium misto ${ }^{(1,3)}$.

Esse artigo é relevante à medida que oferece um material que estimula a enfermagem a ter um pensamento mais crítico e que se torne agente de mudanças em relação ao cuidado, principalmente no tratamento intensivo, onde o cliente necessita de recursos para a manutenção de sua qualidade de vida, com a intenção de projetar uma maior visibilidade para os profissionais da enfermagem no que tange ao cuidado específico prestado a pacientes acometido por delirium.

Tendo em vista o alinhamento da pesquisa, nossa questão de pesquisa é descobrir como o enfermeiro identifica o delirium nos pacientes assistidos por ele na UTI, nas publicações cientificas de enfermagem em saúde, visando quais são as suas construções para a prática de enfermagem. Tendo como objeto de pesquisa evidenciar e discutir as principais características e manifestações do delirium nas UTIs, bem como o enfermeiro pode contribuir para as assistências da equipe de saúde destas UTIs, com base nas evidências apontadas pela literatura científica.

\section{METODOLOGIA}

Trata-se de uma revisão integrativa da literatura, pois permite a síntese de estudos publicados e possibilita conclusões de uma particular área de estudo, além de apontar lacunas do conhecimento que necessitam de novos estudos $^{(4)}$.

As etapas percorridas foram: (1) Elaboração da questão norteadora da pesquisa; (2) Estabelecimento de critérios para inclusão e exclusão dos estudos/amostragem e Busca na literatura; (3) Definição das informações a serem extraídas dos estudos selecionados/ categorização dos estudos; (4)
Avaliação dos estudos incluídos na revisão integrativa; (5) Interpretação dos resultados; (6) Apresentação da revisão/síntese do conhecimento(5). O levantamento bibliográfico foi realizado na base de dados Literatura Latino-Americana e do Caribe em Ciências da Saúde (LILACS), Banco de Dados em Enfermagem (BDENF) e Scientific Electronic Library Online (SciELO), e tendo como questão norteadora: Qual o papel do enfermeiro acerca da identificação do Delirium na UTI? Foram utilizados os seguintes descritores: "delirium", "Unidades de Terapia Intensiva" e "enfermagem". Foi utilizada como estratégia de busca "delirium AND unidades de terapia intensiva AND enfermagem".

Como critérios de inclusão, foram delimitadas as publicações ocorridas entre maio de 2013 a abril de 2018, tendo a amostra sido obtida entre os meses de março e abril de 2018. Analisamos artigos que estivessem disponíveis na Íntegra por meio de acervos eletrônicos, artigos publicados em textos completos e no idioma português. Foram excluídos os artigos duplicados nas bases de dados ou que não atendiam ao objeto de estudo.

Para coleta de dados, utilizou-se um instrumento que contemplava informações relacionadas à identificação do artigo, autor (es), periódico publicado, ano da publicação, características metodológicas do artigo e país da publicação.

Foram identificados no total 20 produções e, a partir dos critérios de inclusão foram selecionados 10 estudos, os quais foram impressos, lidos na íntegra, sendo dispostos e apresentados em tabelas.

Os principais aspectos abordados acerca do delirium na UTI foram categorizados em: O reconhecimento do Delirium, Papel da Enfermagem na Utilização da Escala CAM-ICU e Aplicação nas UTIs e Medidas preventivas que devem ser implementadas pelo en- 
fermeiro para evitar a manifestação do Delirium. Posteriormente, foi realizada a análise temática e a discussão com base na literatura relacionada.

Segue um quadro que elucida o cruzamento descritores com as bases de dados, LILACS, BDENF e SciELO, sendo ' $\mathrm{E}$ ' os 'encontrados' e 'S' os 'selecionados' para o estudo.

Quadro 1. Cruzamento de descritores com bases de dados utilizadas para a composição do artigo. Rio de Janeiro, RJ, Brasil, 2018

\begin{tabular}{cccccccc}
\multirow{2}{*}{ CRUZAMENTO } & \multicolumn{2}{c}{ LILACS } & \multicolumn{3}{c}{ BDENF } & \multicolumn{2}{c}{ SCIELO } \\
\cline { 2 - 8 } & E & Delirium & S & E & S & E & S \\
Delirium and UTI & 86 & 1 & 9 & 2 & 69 & 5 \\
\hline Delirium and UTI and Enfermagem & 13 & 2 & 5 & 1 & 21 & 3 \\
\hline
\end{tabular}

Nota: $\mathrm{E}=$ Encontrados; $\mathrm{S}=$ Selecionados.

\section{RESULTADOS}

Segue abaixo o Quadro 2, que apresenta os artigos selecionados a partir da busca estabelecida na metodologia, de acordo com os critérios de seleção. Os artigos estão distribuídos entre os anos de 2013 e 2017, publicados em diferentes periódicos, destacando-se a Revista Brasileira de Terapia Intensiva com maior quantitativo de artigos selecionados. Tais estudos evidenciaram a necessidade de aprofundar os esforços na compreensão, avaliação e tratamento do Delirium na UTIs do país. Neste quadro, de forma simplificada, os artigos são apresentados de acordo com seu autor, pais, periódico, tipos de estudo e seus respectivos objetivos, resultados e conclusões.

Quadro 2: Disposição dos artigos selecionados de acordo com autores, país, periódico, tipo de estudo, objetivo, resultados e ano de publicação. Rio de Janeiro, RJ, Brasil, 2018

AUTOR/ANOI PERIÓDI- TIPO DE PAÍs

Mesa $\mathrm{P}$, Previgliano IJ,

Altez $\mathrm{S}$, Favretto

$\mathrm{S}$, Orellano

$M$, Lecor $C$, et

al.,2017, Brasil3

Estudo Brasileira de Terapia Intensiva

\section{OBJETIVOS} Revista prospectivo de

Souza RCS, Bersaneti MDR, Siqueira EMP,

2 Meira L, Brumatti DL, Prado NRO, 2017, Brasil12

$\begin{array}{ccc}\text { Mori S, Takeda } & \text { Revista da } & \\ \text { JRT, Carrara } & \text { Escola de } & \text { Corte prospec- } \\ \text { FSA, Cohrs CR, } & \text { enferma- } & \text { tivo. } \\ \text { Zanei SSV, Whi- } & \text { gem da } & \\ \text { taker IY, 2016, } & \text { USP } & \\ \text { Brasil13 } & \end{array}$
delirium. do delirium em uma unidade de terapia intensiva geral e identificar os fatores associados. unidade de terapia intensiva clínico-cirúrgica.
Estabelecer a prevalência

\section{RESULTADOS}

A prevalência de delirium foi de 184 pacientes com delirium em um total de 230 pacientes. 0 subtipo de delirium psicomotor foi hiperativo em 11 pacientes $(6 \%)$, hipoativo em $9(5 \%)$ e misto em 160 (89\%).

$\begin{array}{ccc}\text { Revista } & \text { Relato de } & \text { Narrar a experiência de } \\ \text { Gaúcha de } & \text { que abrangeu } & \begin{array}{c}\text { capacitação de enfermei- } \\ \text { ros para implementação }\end{array} \\ \text { Enferma- } & \begin{array}{c}\text { as etapas de } \\ \text { diagnóstico }\end{array} & \begin{array}{c}\text { da avaliação sistematiza- } \\ \text { gem }\end{array} \\ \text { situacional. } & \text { da do delirium. }\end{array}$

Identificar a incidência de delirium, comparar as características demográficas e clínicas dos pacientes com e sem
Do total de 149 pacientes da amostra, 69 (46,3\%) apresentaram delirium durante a internação na UTI

CONCLUSÕES/ RECOMENDAÇÕES

Em ambas as análises, identificamos que a duração da ventilação mecânica é um preditor de desfechos desfavoráveis.

A experiência demonstrou que essa avaliação possibilita analisar os resultados do processo de trabalho e a transformação consequente das iniciativas no cotidiano.

Elucidou-se um alto índice de delirium na UTI e sua ocorrência associada às idades mais avançadas e o uso de sedativos e analgésicos.

Há uma grande neces-

Identificar a prevalên-

Luna AA, Revista de Entringer AP, Enferma-

4

Silva RCL, 2016, gem da Brasil7
Estudo

transversal retrospectivo. cia do delirium entre pacientes internados em uma unidade de terapia intensiva do Estado do Rio de Janeiro.
Evidenciou-se que somente dois pacientes tiveram o diagnóstico confirmado, com uma prevalência de $2 \%$ do delirium. sidade de ampliação das discussões acerca do uso de protocolos de avaliação para o diagnóstico de delirium entre os profissionais. 


\begin{tabular}{|c|c|c|c|c|c|c|}
\hline 5 & $\begin{array}{l}\text { Ribeiro SCL, } \\
\text { Nascimento } \\
\text { ERP, Lazzari } \\
\text { DD, Jung W, } \\
\text { Boes AA, } \\
\text { Bertoncello } \\
\text { KC, 2015, } \\
\text { Brasil5 }\end{array}$ & $\begin{array}{l}\text { Revista Tex- } \\
\text { to contexto } \\
\text { enfermagem }\end{array}$ & $\begin{array}{l}\text { Qualitativa, } \\
\text { descritiva e } \\
\text { exploratória. }\end{array}$ & $\begin{array}{l}\text { Analisar o conhecimento de } \\
\text { enfermeiros de uma unidade } \\
\text { de terapia intensiva acerca do } \\
\text { delirium no paciente crítico. }\end{array}$ & $\begin{array}{l}\text { Da análise dos dados } \\
\text { emergiram cinco discursos: } \\
\text { sinais e sintomas; uso de } \\
\text { sedativos; contenção física; } \\
\text { ambiente; falta de preparo } \\
\text { profissional. }\end{array}$ & $\begin{array}{l}\text { Aponta-se para a necessi- } \\
\text { dade de educação sobre } \\
\text { o tema e novos estudos } \\
\text { sobre manejo do delirium } \\
\text { para a enfermagem. }\end{array}$ \\
\hline 6 & $\begin{array}{l}\text { Tanaka LM, } \\
\text { Salluh J, } \\
\text { Dal-Pizzol F, } \\
\text { Barreto BB, } \\
\text { Zantieff R, } \\
\text { Tobar E, et } \\
\text { al., 2015, } \\
\text { Brasil11 }\end{array}$ & $\begin{array}{l}\text { Revista } \\
\text { Brasileira } \\
\text { de Terapia } \\
\text { Intensiva }\end{array}$ & $\begin{array}{l}\text { Avaliação Sis- } \\
\text { temática onde } \\
\text { foi utilizado } \\
\text { um questioná- } \\
\text { rio eletrônico } \\
\text { para avaliar } \\
\text { o perfil dos } \\
\text { entrevistados } \\
\text { e das unidades } \\
\text { de terapia } \\
\text { intensiva. }\end{array}$ & $\begin{array}{l}\text { Conduzir um inquérito multi- } \\
\text { nacional com profissionais de } \\
\text { unidades de terapia intensiva } \\
\text { para determinar as práticas } \\
\text { relacionadas à avaliação e ao } \\
\text { manejo do delirium, bem como } \\
\text { as percepções e as atitudes } \\
\text { relacionadas à avaliação e ao } \\
\text { impacto do delirium em pacien- } \\
\text { tes submetidos à ventilação não } \\
\text { invasiva. }\end{array}$ & $\begin{array}{l}\text { Foram analisados } 436 \\
\text { questionários que, em sua } \\
\text { maioria, eram provenientes } \\
\text { do Brasil, seguidos de } \\
\text { Turquia e Itália. }\end{array}$ & $\begin{array}{l}\text { Enfatizou- se a escassez } \\
\text { de esforços direcionados } \\
\text { à avaliação e ao manejo } \\
\text { do delirium no ambiente } \\
\text { da terapia intensiva, em } \\
\text { especial nos pacientes } \\
\text { submetidos à ventilação } \\
\text { não invasiva. }\end{array}$ \\
\hline 7 & $\begin{array}{l}\text { Luna AA, Bridi } \\
\text { AC, Silva RCL, } \\
\text { 2015, Brasil9 }\end{array}$ & $\begin{array}{l}\text { Revista de } \\
\text { Enfermagem } \\
\text { UFPE }\end{array}$ & $\begin{array}{l}\text { Estudo } \\
\text { retrospectivo } \\
\text { transversal. }\end{array}$ & $\begin{array}{l}\text { Identificar a ocorrência de risco } \\
\text { do delirium entre pacientes em } \\
\text { uso de sedativos em uma UTI }\end{array}$ & $\begin{array}{l}\text { Observou- se achados clíni- } \\
\text { cos sugestivos de delirium, } \\
\text { em } 69,53 \% \text {, no entanto, } \\
\text { apenas dois pacientes } \\
\text { diagnosticados. }\end{array}$ & $\begin{array}{l}\text { Os resultados parecem } \\
\text { sugerir de fato o quanto } \\
\text { é comum o subdiagnós- } \\
\text { tico do delirium entre } \\
\text { os pacientes em uso de } \\
\text { sedativos na UTI. }\end{array}$ \\
\hline 8 & $\begin{array}{l}\text { Pincelli EL, } \\
\text { Waters C, } \\
\text { Hupsel ZN, } \\
\text { 2015, Brasil6 }\end{array}$ & $\begin{array}{l}\text { Arq Med } \\
\text { Hosp Fac } \\
\text { Cienc Med } \\
\text { Santa Casa } \\
\text { São Paulo }\end{array}$ & $\begin{array}{l}\text { Estudo } \\
\text { Bibliográfica e } \\
\text { exploratória. }\end{array}$ & $\begin{array}{l}\text { Identificar, em artigos de perió- } \\
\text { dicos, as açõ̃es de enfermagem } \\
\text { na prevenão do delirium. }\end{array}$ & $\begin{array}{l}\text { As ações de enfermagem } \\
\text { para prevenção do delirium } \\
\text { em pacientes internados } \\
\text { em UTI foram organizadas } \\
\text { nos temas: ambiente; } \\
\text { mobilização; higiene do } \\
\text { sono; equipamento e dis- } \\
\text { positivos; família e amigos } \\
\text { e controle fisiológico. }\end{array}$ & $\begin{array}{l}0 \text { tratamento mais efetivo } \\
\text { do delirium ainda é } 0 \\
\text { preventivo. }\end{array}$ \\
\hline 9 & $\begin{array}{l}\text { Faria RSB, } \\
\text { Rui P, 2013, } \\
\text { Brasil8 }\end{array}$ & $\begin{array}{l}\text { Revista } \\
\text { Brasileira } \\
\text { de Terapia } \\
\text { Intensiva }\end{array}$ & $\begin{array}{l}\text { Revisão inte- } \\
\text { grativa. }\end{array}$ & $\begin{array}{l}\text { Analisar fatores de risco, mani- } \\
\text { festações clínicas e abordagens } \\
\text { preventivas e terapêuticas. }\end{array}$ & $\begin{array}{l}\text { Foram revistos os principais } \\
\text { fatores de risco, manifesta- } \\
\text { ções clínicas e abordagens } \\
\text { preventivas e terapêuticas. }\end{array}$ & $\begin{array}{l}\text { Prevenir é melhor que } \\
\text { tratar, por isso é impor- } \\
\text { tante identificar os fatores } \\
\text { de risco, sobretudo os } \\
\text { modificáveis. }\end{array}$ \\
\hline 10 & $\begin{array}{l}\text { Salluh Jl, } \\
\text { Stevens } \\
\text { RD, 2013, } \\
\text { Brasil10 }\end{array}$ & $\begin{array}{l}\text { Revista } \\
\text { Brasileira } \\
\text { de Terapia } \\
\text { Intensiva }\end{array}$ & $\begin{array}{l}\text { Revisão siste- } \\
\text { mática descri- } \\
\text { tiva de escalas } \\
\text { de gradação } \\
\text { de delirium } \\
\text { em pacientes } \\
\text { criticamente } \\
\text { enfermos. }\end{array}$ & $\begin{array}{l}\text { Identificar o delirium por meio } \\
\text { do uso da Escala CAM-ICU e } \\
\text { ICDSC. }\end{array}$ & $\begin{array}{l}\text { Foram identificadas } 6 \\
\text { escalas validadas para } \\
\text { identificação da delirium } \\
\text { em pacientes criticamente } \\
\text { enfermos. }\end{array}$ & $\begin{array}{l}\text { Esforços educacionais e } \\
\text { treinamento de profis- } \\
\text { sionais de saúde, na im- } \\
\text { plantação sistemática de } \\
\text { ferramentas de triagem, } \\
\text { são passos fundamentais } \\
\text { neste processo. }\end{array}$ \\
\hline
\end{tabular}

\section{DISCUSSÃO}

Após uma leitura esmiuçada dos artigos selecionados e coleta de dados, emergiram três categorias temáticas relacionadas ao objeto de estudo: O reconhecimento do Delirium, Papel da Enfermagem na utiliza- ção da Escala CAM-ICU e Aplicação nas UTIs e Medidas preventivas que devem ser implementadas pelo enfermeiro para evitar a manifestação do Delirium.

O reconhecimento do Delirium

O delirium constitui uma alteração neu- ropsiquiátrica aguda ou subaguda que afeta a consciência e, geralmente, está acompanhada por algum grau de distúrbio do estado de despertar. São manifestações desse distúrbio: diminuição da atenção e alterações secundárias das funções cognitivas, 
Gois, J.; Vieira, T.; Lourenco, B.S.; Souza, D.R.S.; Valentim, L;; Reis, L;:Assistência de enfermagem ao paciente com delirium na unidade de terapia intensiva: uma revisão integrativa

entre elas percepção, memória, orientação e raciocínio, além de comprometimento do ciclo sono-vigília e transtornos emocionais (depressão, ansiedade, medo, irritabilidade, euforia e apatia). Manifestações essas que têm início abrupto, apresentando flutuação em intensidade durante o dia e, também, de um dia para o outro ${ }^{(5)}$.

Delirium é considerado como uma manifestação heterogênea e foram descritos três subtipos para melhor caracterizá-los: o hiperativo, que é caracterizado pelo aumento da atividade psicomotora, perturbação das percepções e alucinações; o hipoativo, caracterizado pela desatenção e a diminuição da atividade psicomotora; e misto caracterizado pela flutuação imprevisível dos outros subtipos ${ }^{(6)}$.

Os fatores de risco para delirium podem ser classificados em modificáveis e não modificáveis. Os fatores modificáveis são as possíveis situações do ambiente em que o paciente está inserido, assim como isolamento, contenção física no leito, privação do sono e até mesmo ausência de visitas e a doença aguda no período de internação, como febre, desidratação, alto risco de morte, utilização de sedativos e analgésicos, utilização de dispositivos invasivos, como os tubos e cateteres. E entre aos fatores não modificáveis estão às características do próprio paciente, como idade, gênero e hábitos pessoais, como: tabagismo e abuso de álcool, comorbidades, doenças prévias do sistema nervoso e demência ${ }^{(7)}$.

O ambiente típico de uma UTI representa um fator de risco por si só, devido à ausência de iluminação natural, ausência de relógios, perturbação dos padrões de sono e vigília e pelo isolamento do doente ${ }^{(8)}$.

A sedação representa um fator fundamental para o desenvolvimento de delirium. A literatura demonstra que não é somente a exposição à sedação, mas também o tipo de sedativo e a sedação excessiva podem influenciar no desenvolvimento dessa patologia ${ }^{(7)}$.

Porém, é uma patologia que frequentemente manifesta-se nos pacientes graves internados em UTI devido a fatores de risco, tais como: idade (geralmente em pacientes maiores de 65 anos), uso de ventilação me- cânica, realização de procedimentos invasivos, interrupções dos ciclos de sono, hipertensão arterial sistêmica, etilismo, distúrbios metabólicos, uremia, hipoxemia, anemia, acidose, além de comprometimento visual e auditivo, intervenções cirúrgicas e uso de drogas como a morfina ${ }^{(5)}$.

Existem vários sinônimos para nomear esta síndrome, como: estado confusional agudo, falência cerebral aguda, psicose exógena, encefalopatia metabólica, psicose tóxica, entre outros. Porém, a bibliografia sobre cuidados intensivos acatou recentemente as recomendações da APA e de outros especialistas, para que o termo "delirium" seja usado de forma única para descrever esta síndrome ${ }^{(7)}$.

O delirium é um distúrbio neurológico que tem recebido pouca atenção dos profissionais que trabalham na UTI, principalmente, por não ser a razão primária da internação. Por isso, o conhecimento e educação são a base para saber reconhecer os sintomas, aplicar as intervenções necessárias e minimizar a incidência ${ }^{(5)}$.

O delirium é um distúrbio neurológico frequentemente manifestado em pacientes internados em UTI, porém ainda é subdiagnosticado $^{(9-11)}$. E ainda tem sido associado ao maior tempo de permanência do paciente no hospital, aos custos hospitalares, uso de sedativos e restrições mecânicas, e ao aumento da mortalidade. Sua prevalência na UTI varia de acordo com cada estudo, podendo acometer até $80 \%$ dos pacientes submetidos à ventilação mecânica ${ }^{(9,11,12)}$.

Clinicamente, pode se apresentar na forma hiperativa, hipoativa ou mista. Na forma hiperativa é predominante a agitação, algumas vezes com agressividade, raramente passa despercebida. Na forma hipoativa o paciente apresenta-se com nível de consciência rebaixado, geralmente prostrado e pouco responsivo aos estímulos, sendo assim associada com um pior prognóstico, comparado a forma hipoativa. E na forma mista, ocorre alternância entre as duas formas ${ }^{(6,7)}$. Enfermeiros identificam o paciente com Delirium por meio destas manifestações, tais como, agitação ou apatia e com o fornecimento de informações não coerentes ${ }^{(5)}$. O quadro mais frequente do delirium é a forma hipoativa ${ }^{(7,12)}$, enquanto a forma hiperativa pura é relativamente rara, menor que $5 \%$.

A característica clínica que, geralmente, identifica o doente em delirium é o seu início abrupto, em dias, horas ou até minutos, apresentando flutuação em intensidade e de duração limitada ${ }^{(5)}$. É de suma importância que os profissionais de enfermagem possuam conhecimento adequado acerca dos tipos do Delirium, pois o enfermeiro tem papel fundamental na prevenção do Delirium $^{(13)}$ e na recuperação do paciente, devido ao contato constante com o mesmo, podendo observar, de forma mais apurada, alterações do seu status mental ${ }^{(5)}$.

Estudos mostram que os pacientes mais vulneráveis para o desenvolvimento do Delirium são aqueles com idade acima dos 65 $\operatorname{anos}^{(5,7,8)}$ e maior predominância no sexo masculino $^{(7)}$. Frequentemente, manifesta-se em pacientes internados em UTI devido a fatores de risco, tais como idade $\mathrm{e}^{(5-7,13)}$, uso de ventilação mecânica ${ }^{(9,11-13)}$, realização de procedimentos invasivos ${ }^{(5,7)}$, interrupções dos ciclos de sono ${ }^{(5-7)}$, o uso de medicamentos como a morfina ${ }^{(6-8,13)}$ e sedativos $^{(6,8,13)}$.

Pacientes que desenvolvem o Delirium apresentam resultados negativos na evolução, contribuindo para o aumento do tempo de hospitalização(5,6,11,13), maior possibilidade de ocorrência de eventos ad$\operatorname{versos}^{(6,12,13)}$, maior mortalidade ${ }^{(6,7,10,11,13)}$ e ainda custos hospitalares ${ }^{(6,7,12)}$.

O conhecimento dos enfermeiros sobre os fatores que podem favorecer a ocorrência do delirium é essencial para que realizem o planejamento da assistência de enfermagem $^{(13)}$. O reconhecimento juntamente a uma avaliação física e psíquica do paciente são importantes para um diagnóstico preciso, bem como, um tratamento adequado ${ }^{(6)}$.

Papel da Enfermagem na utilização da Escala CAM-ICU e Aplicação nas UTIs

A equipe de Enfermagem tem grande importância e maior tempo de dedicação à avaliação neurológica diária do paciente de terapia intensiva ${ }^{(2)}$.

Podemos ressaltar também, a importância da atuação do profissional enfer- 
meiro na utilização das escalas para identificação do Delirium, existem diversas escalas e instrumentos para identificação do delirium, porém a mais citada nos artigos e utilizada atualmente é o Método de Avaliação da Confusão Mental na UTI (CAM - ICU), que é uma escala criada especificamente para utilização em UTIs para se diagnosticar o delirium, adaptada para a triagem de pacientes entubados ou com ventilação mecânica, podendo ser realizada por médicos e enfermeiros e ser aplicada uma vez ao dia ou a cada período, dependendo da necessidade do paciente. Esta escala verifica quatro questões: flutuação do estado mental, inatenção, pensamento desorganizado e nível de consciência alterado. E ainda trazem que a sua utilização exige apenas um rápido treinamento da equipe, sendo um formulário de fácil aplicação para o diagnóstico ${ }^{(10-13)}$.

Medidas preventivas que devem ser implementadas pelo enfermeiro para evitar a manifestação do Delirium

$\mathrm{Na}$ abordagem do Delirium, o enfermeiro deve ter um olhar holístico, tendo em vista, mudar seu foco curativo para preventivo, buscando propor intervenções multifatoriais focadas nos fatores de risco, reduzin- do, assim, a sua incidência ${ }^{(5,8,10)}$.

As ações de enfermagem para a prevenção do Delirium em pacientes na UTI incluem seis aspectos: ambiente, mobilização, higiene do sono, equipamento e dispositivos, família e amigos e controle fisiológico ${ }^{(6)}$.

Existe uma série de ações de enfermagem no intuito de prevenir o Delirium, no entanto, são voltadas à melhoria do sono $^{(6,8,10,13)}$, orientação do paciente ${ }^{(6,8)}$, facilitação do acesso de familiares ${ }^{(6,10,13)}$, manejo da dor ${ }^{(5,6,8)}$, oferecimento de estímulos cognitivos, verificação de má nutrição e de déficit sensoriais ${ }^{(6)}$. E a enfermagem ainda tem, também, papel fundamental na avaliação do paciente em terapia sedativa, adequando a melhor forma do seu uso ${ }^{(5,6,8,13)}$.

As avaliações física e psíquica do paciente são importantes na busca de fatores de risco para a prevenção e, ainda, para um diagnóstico preciso e tratamento adequado ${ }^{(6)}$.

\section{CONCLUSÃO}

O trabalho denotou o quão importante é o conhecimento do enfermeiro na identificação do Delirium no paciente a fim de evitar um pior prognóstico, não prolongar a sua estadia no ambiente da UTI, bem como, o aumento dos custos para a insti- tuição onde o paciente está hospitalizado.

Para identificação do Delirium, é necessário que a equipe tenha um olhar diferenciado, em especial o enfermeiro que realiza a maior parte dos cuidados e está diretamente ligado à assistência que é prestada aos pacientes criticamente enfermos.

A dificuldade na identificação da patologia existe, uma vez, que a mesma é subvalorizada e de difícil reconhecimento, principalmente, quando se manifesta na forma hipoativa que é caracterizada por sonolência e apatia, que são manifestações extremamente frequentes no paciente crítico.

Através deste estudo, pode-se concluir que a identificação do Delirium é uma prática complexa e minuciosa, que para ser realizada necessita de conhecimento teórico-científico adequado com a finalidade de evitar agravos ao quadro clínico do paciente e reverter as causas evidentes.

Vale ressaltar que o tratamento não farmacológico da patologia é correspondente à prevenção, sendo a primeira meta a ser implementada pelo enfermeiro para a resolução do quadro de Delirium, visando eliminar os fatores predisponentes e precipitantes, avaliando e tratando prontamente cada causa presumida ou confirmada, retirando as drogas potencialmente prejudiciais.

\section{Referências}

1. Lima MLNM, Moreira RO, Okamoto TO,Amaral JB. Delirium em terapia intensiva: revisão sistemártica. Repositório Institucional da Escola Bahiana de Medicina e Saúde Pública [Internet]. 2016 [citado em março 2018]. Disponível em: http://www7.bahiana.edu.br//jppuil handle/bahiana/752.

2. Cabral JVB, Rocha RT, Gouveia VA. Diagnóstico de Delirium pelo método de avaliação da confusão em unidade de cuidados intensivos. Rev Enferm Atenção Saúde [Intemet]. 2017 [citado em março de 2018]; 6(2):169-178. Disponível em: http://seer.uftm.edu.br/revistaeletronica/index.php/enfer/article/view/2014/pdf.

3. Mesa P, Previgliano IJ, Altez S, Favretto S, Orellano M, Lecor C, Soca A, Ely EW. Delirium em uma unidade de terapia intensiva latino-americana. Estudo prospectivo em coorte em pacientes em ventilação mecânica. Rev Bras Ter Intensiva [Intemet]. 2017 [citado em março de 2018]; 29(3):337-345. Disponivel em: http://ref.scielo.org/wbx34k.

4. Mendes KDS, Silveira RCCP, Galvão CM. Revisão integrativa: método de pesquisa para a incomoração de evidências na saúde e na enfermagem. Texto \& contexto enferm [Internet]. 2008 [citado em março 2018]; 17(4): 758-64. Disponível em: http://www.scielo.br/pdf/tcel v17n4/18.pdf.

5. Ribeiro SCL. Conhecimento de enfermeiros sobre delirium no paciente crítico: discurso do sujeito coletivo. Texto \& contexto enferm [Intemet]. 2015 [citado em março de 2018]; 24(2): 513-20. Disponivel em: http://uww.scielo.br/pdf/tce/N24n2/pt_0104-0707-tce-24-02-00513.pdf.

6. Pincelli $E L$, Waters $C$, Hupsel ZN. Ações de enfermagem na prevenção do delirium em pacientes na Unidade de Terapia Intensiva.Arq. Med. Hosp. Fac. Cienc. Med. Santa Casa São Paulo [Intemet]. 2015 [citado em mar 2018]; 60:131-9. Disponível em: http://uww.fomsantacasasp.edu.br/images/Arquivos_medicos/600/60/AR33.pdf.

7. Luna AA, Bridi AC, Silva RCL. Delirium em Terapia Intensiva - um estudo retrospectivo.
Rev.Enfermagem UFPE. 2015; 9(1):69-75.

8. Faria RSB, Moreno RP. Delirium na unidade de cuidados intensivos: uma realidade subdiagnosticada. Rev. bras. ter. intensiva [Internet]. 2013 [citado 2018 mar 28]; 25(2): 137-147. Disponivel em: http://www.scielo.br/scielo.php?pi$d=$ S0103-507X2013000200012\&script=sci_abstract\&tlng=pt.

9. Luna $A A$, Entringer AP, Silva RCL. Prevalência do subdiagnóstico de delirium entre pacientes internados em unidade de terapia intensiva. Rev enfermagem UERJ [Internet]. 2016 [citado em abril 2018]; 24(1):e6238. Disponivel em: http:/l dx.doi.org/10.12957/reuerj.2016.6238.

10. Salluh JF, Stevens RD. Aumento da conscientização sobre delirium em pacientes criticamente enfermos. Rev. bras. ter. intensiva [Internet]. 2013 [citado em abril 2018]; 25(2): 75-76. Disponivel em: http://www.scielo.br/pdf/rbti/v25n2/v25n2a02.pdf.

11. Tanaka LMS, Salluhll F, Dal-Pizzol F, Barreto BB, Zantieff R, Tobar E, Esquinas A, Quarantini LC, Flores DG. Delirium em pacientes na unidade de terapia intensiva submetidos à ventilação não invasiva: um inquérito multinacional. Rev. bras. ter. Intensiva [Intemet]. 2015 [citado em abril 2018]; 27(4), 360-368. Disponível em :http://www.scielo.br/pdf/rbt//27n4/ 0103-507X-rbti-27-04-0360.pdf.

12. Souza RCS, Bersaneti MDR, Siqueira EMP, Meira L, Brumatti DL, Prado NRO. Capacitação de enfermeiros na utilização de um instrumento de avaliação de delinum. Rev. Gaúcha Enferm. [Internet]. 2017 [citado em abril 2018]; 38(1): e64484. Disponível em: http://www. scielo.br/scielo.php?script=sci_arttext\&pid=S1983-14472017000100801\&lng=en. 13. Mori S, Takeda JRT, Carrara FSA, Cohrs CR, Zanei SSV, Whitaker IY. Incidência e fatores relacionados ao delirium em unidade de terapia intensiva. Rev. esc. enferm. USP [Internet]. 2016 [citado em março 2018]; 50(4):587-593. Disponivel em: http://www.scielo.br/pdf/reeusp/v50n4/pt_0080-6234-reeusp-50-04-0587.pdf. 